required liver biopsy. Of the remainder, 16 (59\%) did not require biopsy because Fibroscan had answered the clinical question and three failed to attend for review. Overall, 53 (80\%) did not require a liver biopsy after Fibroscan was used to determine the presence or absence of advanced fibrosis or cirrhosis.

Conclusion In our cohort, Fibroscan prevented the need for liver biopsy in $80 \%$ of patients. Our main use of the test has been in attempting to identify patients with cirrhosis who might need hepatoma screening. Fibroscan is highly effective in reducing the need for biopsy in such cohorts.

Competing interests None declared.

\section{PTU-086 KLF6 SPLICE VARIANT 1 IN PATIENTS WITH COLORECTAL LIVER METASTASIS CORRELATES WITH A HIGHER CLINICAL RISK SCORE}

doi:10.1136/gutjnl-2012-302514c.86

${ }^{1,2} \mathrm{~K}$ Y D Hui, ${ }^{2,3}$ S Robinson, ${ }^{1} \mathrm{G}$ L Patman, ${ }^{2} \mathrm{D}$ Manas, ${ }^{1,4} \mathrm{H}$ L Reeves, ${ }^{2,3} \mathrm{~S}$ White. ${ }^{1}$ Northern Institute for Cancer Research, Newcastle University, Newcastle upon Tyne, UK; ${ }^{2}$ Department of HPB and Transplant Surgery, Freeman Hospital, Newcastle Upon Tyne, UK; ${ }^{3}$ Institute of Cellular Medicine, Newcastle University, UK; ${ }^{4}$ Department of Hepatology, Freeman Hospital, Newcastle upon Tyne, UK

Introduction In its wild type form KLF6 (KLF6-WT) acts as a tumour suppressor gene whose expression is lost in a variety of human cancers. Conversely, KLF-6 splice variant 1 (KLF6-SV1) is able to function as an oncogene playing an important role in tumour proliferation and invasion. The aim of this study was to determine how the expression of these two KLF6 variants in patients with colorectal liver metastases (CRLM) correlates with an established prognostic scoring system.

Methods Patients undergoing resection of CRLM over a 2-year period were identified for inclusion within this study. Immunohistochemistry was performed using antibodies directed against KLF6WT and KLF6-SV1 on formalin fixed paraffin embedded tumour samples and the proportion of positively stained nuclei for each was determined using an automated computerised image analysis system. Clinical data were collected and Fong's Clinical Risk Score (CRS) was calculated as a marker of patients outcome. Spearmans correlation coefficient was used to determine the relationship between nuclear staining of KLF- 6 and the CRS. Continuous variables were compared with Mann-Whitney U test. A p value $<0.05$ was considered significant

Results 25 patients (male $64 \%$ ) were included in this study with a mean age of 69.2 years (range 52-81). The distribution of CRS were as follows; CRS score $0 \mathrm{n}=2$, CRS score $1 \mathrm{n}=6$, CRS score $2 \mathrm{n}=3$, CRS score $3 \mathrm{n}=10$, CRS score $4 \mathrm{n}=3$, CRS score $5 \mathrm{n}=1$. Nine patients developed recurrence within 1 year of liver resection. All patients remained alive at the end of the study period. There was a positive correlation between KLF6-SV1 and the calculated CRS (Spearman's correlation efficient $=0.481, p=0.015$ ) whereas no such correlation existed with KLF6-WT. In those with a CRS $<3$ the mean proportion of positive nuclear staining for KLF6-SV1 was $5 \%$ as compared to $15 \%$ in those with a CRS $\geq 3$ $(\mathrm{p}<0.05)$

Conclusion In this preliminary study, high expression of KLF6-SV1 in patients with CRLM correlates with a higher CRS. Further longterm follow-up data are required to determine what affect this has on prognosis and overall patient survival.

Competing interests K Y D Hui: Grant/Research Support from: Cancer Research UK, the Dowager Countess Eleanor Peel Trust, S Robinson: None declared, G Patman: None declared, D Manas: None declared, H Reeves: None declared, S White: None declared.

\section{PTU-087 MANAGEMENT OF PYOGENIC LIVER ABSCESS: AN 11-YEAR RETROSPECTIVE STUDY OF PRACTICE AT A METROPOLITAN HOSPITAL AND DEVELOPMENT OF LOCAL GUIDELINES}

doi:10.1136/gutjnl-2012-302514c.87

${ }^{1} \mathrm{M}$ E B Fitzpatrick, ${ }^{*} \mathrm{~S}$ Pomfret, ${ }^{1} \mathrm{M}$ Foxton, ${ }^{2} \mathrm{~S}$ Portsmore. 'Department of Gastroenterology, Chelsea and Westminster Hospital, London, UK; ${ }^{2}$ Department of Acute Medicine, Chelsea and Westminster Hospital, London, UK

Introduction Pyogenic liver abscess (PLA) is an uncommon condition associated with considerable morbidity and mortality despite modern treatment. Patients have prolonged lengths of stay, require repeated imaging and procedures, and frequently develop significant complications. We audited the burden of PLA and the outcomes of treatment in Chelsea and Westminster Hospital, London. We used our data to develop local guidelines for PLA, incorporating information from previous case series and a review of the literature.

Methods Between January 2000 and September 2011, 41 patients had a correctly coded diagnosis of PLA. Electronic or paper medical records were available for 40 patients. We collected anonymised information regarding the medical history, investigations and clinical course.

Results 40 patients ( $78 \%$ male, mean age 54 years, $70 \%$ caucasian) were treated for acute PLA, with no in-hospital deaths. Mean length of stay was 19.3 days (CI 15.1 to 23.4 days). Presentations were nonspecific, and the diagnosis rarely considered on admission. Blood results in most patients on admission demonstrated a characteristic pattern of hypoalbuminaemia, high CRP, and non-specific liver dysfunction. $61.1 \%$ of patients were septic on admission. In general, appropriate imaging was arranged early in the admission. Diagnosis of PLA was made with CT (54\%) or ultrasound $(46 \%) .75 \%$ of abscesses were found in the right lobe, with a mean maximum diameter of $6.5 \mathrm{~cm}$ (SD $2.4 \mathrm{~cm}$ ). $48 \%$ of patients had multiple abscesses, and $40 \%$ had signs of loculation on imaging. $78 \%$ of abscesses were larger than $5 \mathrm{~cm}$. $68 \%$ of abscesses were aspirated or drained, with three patients requiring repeated intervention, and one referred to a hepatobiliary unit. Length of stay was longer in older patients $(p<0.05)$, those requiring drainage (23.8 vs 11.3 days, $\mathrm{p}<0.001)$ and those with complications (28 vs 11.8 days, $\mathrm{p}<0.001$ ). Abscess size was not related to length of stay or need for drainage. Decisions regarding antibiotics and drainage varied between clinicians. Severe complications developed in $50 \%$ of patients and included venous thrombosis $(n=4)$ and need for ITU support $(n=4)$. Resolution was typical, with no in-hospital mortality. Underlying causes for PLA included abdominal malignancy $(n=4)$, appendicitis $(n=2)$ and diabetes mellitus $(n=5)$. Often no cause was found, and screening for causes with MRCP and colonoscopy was not universal. Conclusion Our study describes a large UK cohort of patients with PLA, with a lower mortality than described in other case series, and good outcomes without drainage in selected patients with large abscesses. Our cohort showed considerable variation in PLA treatment, and in response we have developed PLA management guidelines.

Competing interests None declared.

\section{PTU-088 THE MICROFLORA OF BILE AFTER INTRAOPERATIVE ASPIRATION OF THE GALLBLADDER DURING CHOLECYSTECTOMY FOR ACUTE CHOLECYSTITIS}

doi:10.1136/gutjnl-2012-302514c.88

M S J Wilson, ${ }^{*}$ K Seymour. General Surgery, North Tyneside General Hospital, North Shields, UK

Introduction Emergency cholecystectomy for the management of acute cholecystitis is now common. In order to facilitate this 
procedure it can be helpful to decompress the gallbladder by aspirating its contents. The benefit of gallbladder aspiration in the elective setting is however unclear. ${ }^{1}{ }^{2}$ It is important to be aware of the likely microflora of bile in patients undergoing emergency cholecystectomy to facilitate the use of appropriate targeted antibiotics. The aim of this study was to establish the prevalence of intraoperative gallbladder aspiration during acute cholecystectomy and to determine the microflora after microscopy and culture.

Methods A retrospective analysis of patients who underwent emergency cholecystectomy for acute cholecystitis over an 18month period (July 2010 to January 2012) identified from PAS data. Cross referencing with microbiology electronic database for microscopy and culture findings from gallbladder aspiration samples.

Results 124 patients (36 male, 88 female, age range 18-90 years) underwent cholecystectomy during the study period. 29 (23.4\%) patients underwent intraoperative aspiration of gallbladder contents, of which $20(69.0 \%)$ had no organisms seen at microscopy and $14(48.3 \%)$ grew no organisms after incubation in culture medium. Abstract PTU-088 table 1 outlines the organisms isolated in the remaining 15 patients; four grew an isolated organism and 11 grew more than one organism and also details the antibiotic profile following culture.

Abstract PTU-088 Table 1 Microorganisms isolated after culture of gallbladder aspirates and antibiotic profile ( $S=$ sensitive, $R=$ resistant)

\begin{tabular}{|c|c|c|c|c|c|c|c|c|c|}
\hline & \multirow[b]{3}{*}{ Frequency } & \multicolumn{8}{|c|}{ Antibiotic } \\
\hline & & \multicolumn{2}{|c|}{ Amoxicillin } & \multicolumn{2}{|c|}{ Augmentin } & \multicolumn{2}{|c|}{ Tazocin } & \multicolumn{2}{|c|}{ Gentamicin } \\
\hline & & $\mathbf{S}$ & $\mathbf{R}$ & $\mathbf{S}$ & $\mathbf{R}$ & $\mathbf{S}$ & $\mathbf{R}$ & $\mathbf{S}$ & $\mathbf{R}$ \\
\hline E coli & 15 & 2 & 10 & 8 & 5 & 12 & 2 & 6 & 0 \\
\hline Klebsiella pneumonia & 4 & 0 & 4 & 5 & 0 & 5 & 0 & 3 & 0 \\
\hline Enterococcus faecalls & 3 & 3 & 0 & 0 & 0 & 0 & 0 & 0 & 0 \\
\hline Enterobacter cloacae & 2 & 0 & 1 & 0 & 2 & 2 & 0 & 0 & 0 \\
\hline
\end{tabular}

Conclusion In order to facilitate emergency cholecystectomy for acute cholecystectomy it is often necessary to decompress the gallbladder by aspirating its contents. In our case series this was necessary in $23 \%$ of patients. Almost half of aspirates were found to be sterile. In the remainder, the most common organism isolated after culture is Escherichia coli, whch is usually resistant to amoxicillin, but sensitive to tazocin. However, the sensitivity of $E$ coli to augmentin is less clear and surgeons should be aware of this when initiating antibiotic prophylaxis for acute cholecystitis

Competing interests None declared.

\section{REFERENCES}

1. Ezer A, Nursal TZ, Colakoglu T, et al. The impact of gallbladder aspiration during elective laparoscopic cholecystectomy: a prospective randomized study. AJS 2008; 196:456-9

2. Calik A, Topaloglu S, Topcu S, et al. Routine intraoperative aspiration of gallbladder during laparoscopic cholecystectomy. Surg Endosc 2007;21:1578-81.

\section{PTU-089 NATIONAL STUDY OF OUTCOMES FOR CHOLANGIOCARCINOMA PATIENTS FOLLOWING BILIARY ENDOSCOPY: LINKAGE ANALYSIS OF ADMINISTRATIVE DATA FOR ENGLISH HOSPITALS (2006-2008)}

doi:10.1136/gutjnl-2012-302514c.89

${ }^{1} \mathrm{~N}$ Polavarapu, ${ }^{*} \mathrm{~K}$ Bowering, ${ }^{2} \mathrm{E}$ Thompson, ${ }^{1} \mathrm{R}$ Sturgess, ${ }^{2} \mathrm{M}$ Pearson, ${ }^{2,3} \mathrm{~K}$ Bodger. ${ }^{1}$ Digestive Diseases Centre, Aintree University Hospital, Liverpool, UK; ${ }^{2}$ Aintree Health Outcomes Partnership, University of Liverpool, Liverpool, UK; ${ }^{3}$ Department of Gastroenterology, Institute of Translational Medicine, University of Liverpool, Liverpool, UK

Introduction Cholangiocarcinoma (CC) is a rare and challenging cancer with poor prognosis and low operative rate. Early successful biliary drainage is a key determinant of outcome and ERCP is the primary modality. It is unclear whether current care organisation for CC is optimal. We report a national study aimed at describing outcomes for all patients undergoing ERCP for CC in English hospitals and volumes at cancer networks and institutions.

Methods We built on linkage methods applied to overall ERCP mortality ${ }^{1}$ to develop new techniques to map the entire pathway of hospital care for incident cases of CC. 2 years of Hospital Episode Statistics (HES) data were merged (2006-2008) and admissions screened for CC diagnosis. To identify a 1-year incident cohort of CC, we selected only patients with first cancer coding in middle year (October-September), then extracted all admissions within 6 months (before and after) of first cancer coding, ordered chronologically, screened for ERCP, radiological intervention (PTC) and major surgery codes. Identified first and subsequent procedure dates, admission diagnoses and co-morbidity. Linkage to death registry for death date. Cases allocated to cancer networks using provider codes. Results Nationally, 1211 CC patients underwent ERCP with mean age (SD) of $\mathbf{7 2}$ (12) years and 623 male (51.4\%). First ERCP was performed during an acute (emergency) hospitalisation in 690 cases (57\%). ERCP case volumes for CC ranged from 7 to 79 patients per Cancer Network and $1-57$ patients per Trust ( $\mathrm{n}=146$ institutions). Outcomes (post-first ERCP): Mortality: 7 day [40 (3.3\%)]; 30 day: 172 (14.2\%); 365 day: 781 (64.5\%). Emergency readmission: 7 day: 110 (9.1\%); 30 day: 252 (20.8\%). Additional PTC $213(\mathbf{1 7 . 6} \%)$ with poorer $365 \mathrm{~d}$ survival in those needing both (ERCP alone: $64.5 \%$ vs ERCP+PTC: $73 \%, p=0.013$, non-surgical cases only). Patients requiring first ERCP during an acute hospitalisation had poorer prognosis than those on elective pathway (Log rank, $p<0.001$ ). 365 day mortality for surgical $\mathbf{4 2 . 4 \%}$ vs non-surgical $\mathbf{6 6 . 2 \%}(\mathrm{p}<0.001)$.

Conclusion First endoscopic intervention for this rare form of cancer is undertaken in most English hospitals, often during acute hospitalisation. There is wide variation in institutional case load. These data provide a potential tool for exploring variation in relation to local or network service provision and organisation.

Competing interests None declared.

\section{REFERENCE}

1. Bodger K, Bowering K, Sarkar S, et al. All-cause mortality after first ERCP in England: clinically guided analysis of hospital episode statistics with linkage to registry of death. Gastrointest Endosc 2011;74:825-33.

\section{PTU-090 TYPE 2 DIABETES AS A POSITIVE RISK FACTOR IN THE AETIOLOGY OF CHOLANGIOCARCINOMA: A CASE-CONTROL STUDY IN TWO UK CENTRES}

doi:10.1136/gutjnl-2012-302514c.90

${ }^{1} \mathrm{~N}$ Burr, ${ }^{2} \mathrm{R}$ Talboys, ${ }^{3} \mathrm{~S}$ Savva, ${ }^{2} \mathrm{M}$ P Lewis, ${ }^{1} \mathrm{~S}$ Rushbrook, ${ }^{1} \mathrm{M}$ Phillips, ${ }^{2} \mathrm{M}$ Rhodes, ${ }^{3} \mathrm{R}$ Robinson, ${ }^{4} \mathrm{M}$ Metcalfe, ${ }^{4} \mathrm{~A}$ Dennison, ${ }^{5} \mathrm{~A}$ Clark, ${ }^{1} \mathrm{~A}$ Hart. ${ }^{1}$ Department of Gastroenterology, Norfolk and Norwich University Hospital, Norwich, UK; ${ }^{2}$ Department of General Surgery, Norfolk and Norwich University Hospital, Norwich, UK; ${ }^{3}$ Gastroenterology, Leicester General Hospital, Leicester, UK; ${ }^{4}$ General Surgery, Leicester General Hospital, Leicester, UK; ${ }^{5}$ Norwich Medical School, Norwich, UK

Introduction The incidence of cholangiocarcinoma has increased worldwide with the mortality still remaining high. The aetiology in Western populations is largely unknown, but diabetes may be involved by influencing the neoplastic process via hyperinsulinaemia and the stimulation of the IGF-1 axis. As there are few population based studies looking at this, the aim of this study was to investigate if there is a positive association between type 2 diabetes and the development of cholangiocarcinoma in two centres in the UK. As oral hypoglycaemics may have anti-cancer properties, these drugs were considered when assessing the effect of diabetes. 